Differential realization of pseudo-Hermiticity: A quantum mechanical analog of Einstein's field equation

Ali Mostafazadeh

Citation: Journal of Mathematical Physics 47, 072103 (2006); doi: 10.1063/1.2212668

View online: http://dx.doi.org/10.1063/1.2212668

View Table of Contents: http://aip.scitation.org/toc/jmp/47/7

Published by the American Institute of Physics

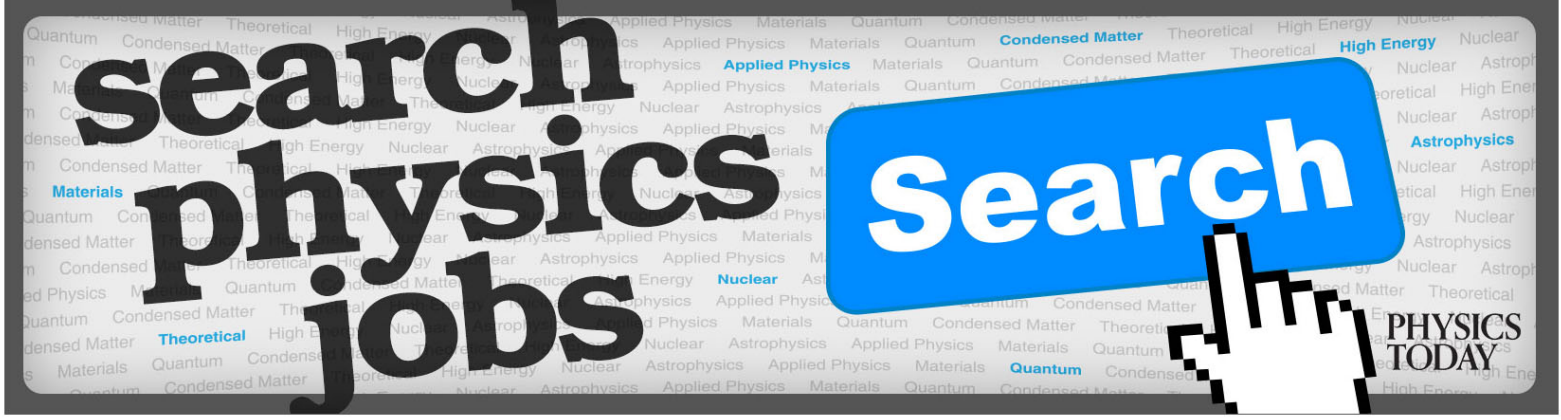




\title{
Differential realization of pseudo-Hermiticity: A quantum mechanical analog of Einstein's field equation
}

\author{
Ali Mostafazadeh ${ }^{\text {a) }}$ \\ Department of Mathematics, Koç University, 34450 Sariyer, Istanbul, Turkey
}

(Received 10 February 2006; accepted 16 May 2006; published online 7 July 2006)

\begin{abstract}
For a given pseudo-Hermitian Hamiltonian of the standard form: $H=p^{2} / 2 m+v(x)$, we reduce the problem of finding the most general (pseudo-)metric operator $\eta$ satisfying $H^{\dagger}=\eta H \eta^{-1}$ to the solution of a differential equation. If the configuration space is $\mathbb{R}$, this is a Klein-Gordon equation with a nonconstant mass term. We obtain a general series solution of this equation that involves a pair of arbitrary functions. These characterize the arbitrariness in the choice of $\eta$. We apply our general results to calculate $\eta$ for the $\mathcal{P} \mathcal{T}$-symmetric square well, an imaginary scattering potential, and a class of imaginary delta-function potentials. For the first two systems, our method reproduces the known results in a straightforward and extremely efficient manner. For all these systems we obtain the most general $\eta$ up to second-order terms in the coupling constants. (C) 2006 American Institute of Physics.
\end{abstract}

[DOI: $10.1063 / 1.2212668]$

\section{INTRODUCTION}

The key aspect of the general theory of relativity (GR) that distinguishes it from other wellestablished physical theories is that in GR the very geometry of the spacetime, which is the arena of physical reality in classical physics, is itself a dynamical quantity. In contrast, in quantum mechanics (QM), the geometry of the Hilbert space, which plays a similar role as the spacetime does in classical physics, is an absolute entity. This is to some extent dictated by the well-known mathematical fact that all separable Hilbert spaces are unitary equivalent. For the past 75 years or so, this equivalence has been used to justify the absolutism associated with the convention of fixing the (inner product of the) Hilbert space from the outset. This is actually quite surprising, for the existence of an equivalence relation in a theory is clearly an evidence of the presence of a freedom in its formulation. In the case of QM, this is the freedom to choose the inner product of the Hilbert space, a freedom that has been left unused until recently. ${ }^{1,2}$

In Ref. 2, we have investigated the consequences of promoting the inner product of the Hilbert space into a degree of freedom. This revealed certain similarities between QM and GR and led to some interesting observations such as a direct link between geometric phases and the geometry of the Hilbert space and a new root to a certain nonlinear generalization of QM. In the present paper, we derive and examine a differential equation that includes among its solutions all possible choices of the inner product for a given physical system. This is the quantum mechanical analog of Einstein's field equation. (The same way Einstein's equation does not generally restrict the metric tensor to have a particular signature, the above-mentioned equation does not restrict its solutions to correspond to positive-definite metric operators.) For a system having $\mathbb{R}$ as its configuration space we obtain a series solution of this equation that involves two functional degrees of freedom. These signify the arbitrariness in the choice of the (pseudo-)metric operator. Our approach allows a more direct way of addressing some of the basic practical problems arising in

\footnotetext{
${ }^{a)}$ Electronic mail: amostafazadeh@ku.edu.tr
} 
the application of quasi- and pseudo-Hermitian quantum mechanics. ${ }^{1,3-13}$ In particular it provides an extremely powerful technical tool for the perturbative calculation of the (pseudo-)metric operators for various toy models.

\section{DIFFERENTIAL REPRESENTATION OF PSEUDO-HERMITICITY}

Consider a physical system described by a separable Hilbert space $\mathcal{H}$ and a pseudo-Hermitian Hamiltonian operator $H: \mathcal{H} \rightarrow \mathcal{H}$. Let $\mathcal{M}$ denote the set of all linear invertible Hermitian operators $\eta: \mathcal{H} \rightarrow \mathcal{H}$, then by definition ${ }^{14}$ the pseudo-Hermiticity of $H$ means that $\mathcal{M}_{H}:=\left\{\eta \in \mathcal{M} \mid H^{\dagger}\right.$ $\left.=\eta H \eta^{-1}\right\}$ is a nonempty subset of $\mathcal{M}$. The elements of $\mathcal{M}$ are called pseudo-metric operators, for they may be used to define a pseudo-inner product (a nondegenerate sesquilinear form ${ }^{15}$ ) $\langle\cdot \mid \cdot\rangle_{\eta}$ $:=\langle\cdot \mid \eta \cdot\rangle$ on $\mathcal{H}$, where $\langle\cdot \mid \cdot\rangle$ denotes the defining inner product of $\mathcal{H}$. (Strictly speaking, $\langle\cdot \mid \cdot\rangle_{\eta}$ is a nondegenerate sesquilinear form defined on the domain of $\eta$ ).

The Hamiltonian $H$ is Hermitian with respect to $\langle\cdot \mid \cdot\rangle_{\eta}$ for all $\eta \in \mathcal{M}_{H \cdot}{ }^{14,16}$ If $\mathcal{M}_{H}$ includes a positive-definite element, i.e., a metric operator, $\eta_{+}$, then $H$ is Hermitian with respect to the positive-definite inner product $\langle\cdot \mid \cdot\rangle_{\eta_{+}}$. This implies that $H$ is diagonalizable and has a real spectrum. The converse of this statement holds true at least for the case that the spectrum of $H$ is discrete, i.e., if $H$ is diagonalizable and has a real spectrum then $\mathcal{M}_{H}$ includes a positive-definite element and equivalently $H$ is Hermitian with respect to a positive-definite inner product. ${ }^{17,18}$ Furthermore, in this case one can show that $H$ is necessarily quasi-Hermitian, i.e., it may be mapped to a Hermitian Hamiltonian $h: \mathcal{H} \rightarrow \mathcal{H}$ via a similarity transformation, $H=\rho^{-1} h \rho .{ }^{17,18}$ This and only this class of Hamiltonians are capable of supporting a unitary time evolution in an associated physical Hilbert space. The latter is defined by endowing $\mathcal{H}$ with the inner product $\langle\cdot \mid \cdot\rangle_{\eta_{+}}$and will be denoted by $\mathcal{H}_{\eta_{+} \cdot} \cdot 4,5,7,9,19$

An important fact about this construction is that $\eta_{+}$is not unique. Different choices for $\eta_{+}$ yield kinematically distinct quantum systems that nevertheless share the same dynamical structure. The quantum mechanical analog of the principle of general covariance of GR is the physical (unitary) equivalence of quantum systems $\left(\mathcal{H}_{\eta_{+}}, H\right){ }^{2}$ The metric operators $\eta_{+}$and more generally pseudo-metric operators $\eta$ are linked to and consequently determined by the Hamiltonian $H$ via the pseudo-Hermiticity condition

$$
H^{\dagger}=\eta H \eta^{-1}
$$

The same way Einstein's field equation links the metric tensor to the energy-momentum tensor, (1) links the pseudo-metric operator to the Hamiltonian. The resemblance may be made more pronounced for a Hamiltonian of the standard form,

$$
H=\frac{\vec{p}^{2}}{2 m}+v(\vec{x}),
$$

that acts in $L^{2}\left(\mathbb{R}^{n}\right)$. Applying both sides of (1) on $\eta$, substituting (2), and representing the resulting equation in the $\vec{x}$-basis, we find

$$
\left(-\nabla_{x}^{2}+\nabla_{y}^{2}+\frac{2 m}{\hbar^{2}}\left[v(\vec{x})^{*}-v(\vec{y})\right]\right) \eta(\vec{x}, \vec{y})=0,
$$

where $\nabla_{u}^{2}:=\sum_{j=1}^{n} \partial^{2} / \partial u_{j}^{2}$ for $u=x, y$ and $\eta(\vec{x}, \vec{y}):=\langle\vec{x}|\eta| \vec{y}\rangle$. For $n=1$, this is a Klein-Gordon equation with a variable mass term,

$$
\left[-\partial_{x}^{2}+\partial_{y}^{2}+\mu^{2}(x, y)\right] \eta(x, y)=0, \quad \mu^{2}(x, y):=\frac{2 m}{\hbar^{2}}\left[v(x)^{*}-v(y)\right]
$$

According to (3) if $\eta(\vec{x}, \vec{y})$ is a solution, then so is $\eta(\vec{y}, \vec{x})^{*}$. The pseudo-metric operators $\eta$ $\in \mathcal{M}_{H}$ correspond to solutions that satisfy 


$$
\eta(\vec{x}, \vec{y})^{*}=\eta(\vec{y}, \vec{x}) .
$$

Note that even for non-pseudo-Hermitian Hamiltonians of the form (2), Eq. (3) admit solutions. However, these solutions fail to satisfy either the Hermiticity requirement (5) or the invertibility condition:

$$
\int_{\mathbb{R}^{n}} \mathrm{~d}^{n} \vec{y} \eta(\vec{x}, \vec{y}) \psi(\vec{y})=0 \text { implies } \psi=0 .
$$

If $\mathcal{M}_{H}$ happens to include positive-definite elements $\eta_{+}$, then these elements correspond to the solutions $\eta_{+}(\vec{x}, \vec{y})$ of (3) that in addition to (5) satisfy

$$
\int_{\mathbb{R}^{n}} \mathrm{~d}^{n} \vec{x} \int_{\mathbb{R}^{n}} \mathrm{~d}^{n} \vec{y} \psi(\vec{x})^{*} \eta_{+}(\vec{x}, \vec{y}) \psi(\vec{y})>0 \text { for } \psi \neq 0 .
$$

The fact that for a diagonalizable Hamiltonian with a real and discrete spectrum such solutions exist is a consequence of the spectral theorems given in Refs. 17 and 18. Indeed if $\left\{\psi_{n}, \phi_{n}\right\}$ is a biorthonormal system associated with $H$, i.e., $H \psi_{n}=E_{n} \psi_{n}, H^{\dagger} \phi_{n}=E_{n} \phi_{n},\left\langle\psi_{n} \mid \phi_{m}\right\rangle=\delta_{m n}$, then

$$
\eta_{+}(\vec{x}, \vec{y})=\sum_{n} \phi_{n}(\vec{x}) \phi_{n}(\vec{y})^{*}
$$

is a solution of (3) that satisfies both (5) and (7). As shown in Refs. 20 and 21, in this case the most general (positive-definite) metric operator has the form $A^{\dagger} \eta_{+} A$, where $A$ is invertible and commutes with $H$. The latter corresponds to a solution of (3) that is of the form

$$
\eta^{\prime}{ }_{+}(\vec{x}, \vec{y})=\sum_{n} \int_{\mathbb{R}^{n}} \mathrm{~d}^{n} \vec{u} \int_{\mathbb{R}^{n}} \mathrm{~d}^{n} \vec{v} A(\vec{u}, \vec{x})^{*} \phi_{n}(\vec{u}) \phi_{n}(\vec{v})^{*} A(\vec{v}, \vec{y}),
$$

where $A(\vec{x}, \vec{y})$ satisfies

$$
\left(-\nabla_{x}^{2}+\nabla_{y}^{2}+\frac{2 m}{\hbar^{2}}[v(\vec{x})-v(\vec{y})]\right) A(\vec{x}, \vec{y})=0,
$$

and

$$
\int_{\mathbb{R}^{n}} \mathrm{~d}^{n} \vec{y} A(\vec{x}, \vec{y}) \psi(\vec{y})=0 \text { implies } \psi=0 .
$$

For a real-valued potential $v, \delta(\vec{x}-\vec{y})$ is a solution of (3). It corresponds to the choice $\eta=I$, where $I$ is the identity operator acting in $\mathcal{H}=L^{2}(\mathbb{R})$. This is consistent with the Hermiticity of $H$. For a $\mathcal{P} \mathcal{T}$-symmetric potential $v$ that satisfies $v(-\vec{x})=v(\vec{x})^{*}, \delta(\vec{x}+\vec{y})$ is a solution of (3). This is a manifestation of $\mathcal{P}$-pseudo-Hermiticity of the Hamiltonian, ${ }^{14}$ for $\langle\vec{x}|\mathcal{P}| \vec{y}\rangle=\delta(\vec{x}+\vec{y})$.

For $n>1$, (3) is an ultrahyperbolic equation with quite peculiar properties. ${ }^{22}$ We will therefore focus our attention on the case $n=1$. Our main purpose is to obtain the general solution of (3) without having to resort to its spectral decomposition (8). This is mainly because of the difficulties with summing the series in (8) or evaluating the integrals that replace the latter whenever the spectrum becomes continuous. ${ }^{7}$

In Ref. 9, we have pursued a similar approach to construct the most general $\eta_{+}$for the imaginary cubic potential $v=i \varepsilon x^{3}$ in some low orders of perturbation theory. The approach of Ref. 9 applies to any (preferably imaginary) potential with a real spectrum. It yields an infinite system of iteratively decoupled partial differential equations whose solution provides the contributions to $\eta_{+}$in various orders of the perturbation theory. Although these equations have the same structure, at each order one must compute their nonhomogeneous term and solve them separately. In contrast, in the present paper, we obtain a single differential equation satisfied by $\eta$, namely (4), 
that applies for an arbitrary potential $v(x)$ rendering the Hamiltonian pseudo-Hermitian. An important advantage of the approach of the present paper over that of Ref. 9 is that in view of the simple structure of (4), we are able to offer (as discussed in Sec. III) a general scheme for constructing a series solution of this equation. This solution involves two arbitrary functions that provide an explicit characterization of the arbitrariness in the choice of $\eta$.

Another recent application of the powerful machinery of differential equations to compute (pseudo-)metric operators is due to Scholtz and Geyer. ${ }^{10}$ These authors obtain a phase-space representation of the (pseudo-)metric operators $\eta$. They use the Moyal product techniques to deal with the difficult factor-ordering problems that arise in this representation. The following are the main differences between the method of Ref. 10 and the one presented in the present paper.

- The method of Ref. 10 leads to an equation for $\eta$ that is a differential equation ${ }^{11}$ provided that $v(x)$ is a polynomial potential. Even for a polynomial potential the general character and in particular the order of this differential equation depends on the structure of $v(x)$ and its degree. In contrast, in the present paper we offer a universal differential equation, namely (4), that applies for polynomial as well as nonpolynomial potentials, and has the same simple structure for all potentials. It is this appealing property that allows us to treat the well-known toy models of Sec. IV. The application of the method of Ref. 10 to these models yields pseudo-differential equations (differential equations of infinite order) whose solution is extremely difficult if not impossible.

- Suppose $v$ is a polynomial potential, so that the method of Ref. 10 yields a differential equation, and suppose that one is able to solve this equation. Then one obtains an explicit expression for $\eta$ in terms of the operators $x$ and $p$ that involves a number of arbitrary functions. The condition that $\eta$ be Hermitian must be imposed to fix some of these functions. This is done by adopting a set of appropriate boundary conditions. ${ }^{10}$ (The author is unaware of a systematic method of selecting the boundary conditions that achieve this purpose.) In contrast, our method yields an expression for $\eta(x, y)$ that satisfies the Hermiticity condition $\eta(x, y)^{*}=\eta(y, x)$ manifestly and specifies a unique Hermitian $\eta$ according to

$$
(\eta \psi)(x)=\int_{\mathbb{R}} \mathrm{d} y \eta(x, y) \psi(y) .
$$

- It achieves this without making use of the Moyal product or having to select certain boundary conditions that ensures the Hermiticity of $\eta$. Its successful application, however, does not yield an explicit expression for $\eta$ in terms of $x$ and $p$. As explained in Ref. 9, the latter may be obtained by Fourier transforming $\eta(x, y)=\langle x|\eta| y\rangle$ over $y$ to obtain $\langle x|\eta| p\rangle$ and arranging the terms in the expression for $\sqrt{2 \pi \hbar} e^{-i x p / \hbar}\langle x|\eta| p\rangle$ in such a way that $x$ 's are placed to the left of $p$ 's. This is how the issue of ordering of factors is addressed in this construction.

A common feature of both methods is that solving the associated differential equations yields generally non-positive-definite pseudo-metric operators. The (positive-definite) metric operators $\eta_{+}$, if they exist, correspond to certain special solutions that are to be identified using different means. [The construction of $\eta$ given here may be supplemented with the procedure proposed in Ref. 10 for selecting the positive-definite metric operators $\eta_{+}$among $\eta$ 's. This is expected to be a difficult task in practice, and we will not pursue it here. We suffice to point out that given $\eta(x, y)$ we can obtain an expression for $\eta$ in terms of $x$ and $p$ as outlined in Ref. 9. This allows for making direct contact with the approaches of Refs. 10 and 11].

\section{SERIES EXPANSION FOR $\eta(x, y)$}

We begin our analysis by expressing (4) in the form

$$
\left(-\partial_{x}^{2}+\partial_{y}^{2}\right) \eta(x, y)=f(x, y)-f(y, x)^{*},
$$

where 


$$
f(x, y):=\frac{2 m}{\hbar^{2}} v(y) \eta(x, y) .
$$

We note that for a Hermitian $\eta,(12)$ is equivalent to

$$
\begin{aligned}
& \eta(x, y)=\chi(x, y)+\chi(y, x)^{*}, \\
& \left(-\partial_{x}^{2}+\partial_{y}^{2}\right) \chi(x, y)=f(x, y) .
\end{aligned}
$$

Next, we recall that the general solution of the wave equation $\left(-\partial_{x}^{2}+\partial_{y}^{2}\right) u(x, y)=0$ is given by

$$
u(x, y)=u_{+}(x-y)+u_{-}(x+y)
$$

where $u_{ \pm}: \mathbb{R} \rightarrow \mathbb{C}$ are a pair of arbitrary twice-differentiable functions (or distributions). Consequently, the general solution of (12) has the form

$$
\eta(x, y)=u_{+}(x-y)+u_{-}(x+y)+\chi_{p}(x, y)+\chi_{p}(y, x)^{*},
$$

where $u_{ \pm}$satisfy $u_{ \pm}(x)^{*}=u_{ \pm}(\mp x)$ and $\chi_{p}(x, y)$ is a particular solution of (15). The latter is a nonhomogeneous wave equation in $1+1$ dimensions. It admits a particular solution that in view of (13) takes the form

$$
\chi_{p}(x, y)=\frac{m}{\hbar^{2}} \int^{y} \mathrm{~d} r \int_{x-y+r}^{x+y-r} \mathrm{~d} s v(r) \eta(s, r) .
$$

Combining (16)-(18), we find

$$
\eta(x, y)=u(x, y)+\mathcal{K} \eta(x, y),
$$

where $\mathcal{K}$ is the integral operator defined by

$$
\begin{aligned}
\mathcal{K} \eta(x, y) & :=\frac{m}{\hbar^{2}}\left[\int^{y} \mathrm{~d} r \int_{x-y+r}^{x+y-r} \mathrm{~d} s v(r) \eta(s, r)+\int^{x} \mathrm{~d} r \int_{-x+y+r}^{x+y-r} \mathrm{~d} s v^{*}(r) \eta(s, r)^{*}\right] \\
& =\frac{m}{\hbar^{2}}\left[\int^{y} \mathrm{~d} r \int_{x-y+r}^{x+y-r} \mathrm{~d} s v(r) \eta(s, r)+\int^{x} \mathrm{~d} s \int_{-x+y+s}^{x+y-s} \mathrm{~d} r v(s)^{*} \eta(s, r)\right] .
\end{aligned}
$$

In view of the analogy with the derivation of the Lippmann-Schwinger equation, ${ }^{23}$ it is not difficult to see that (19) admits the following general series solution:

$$
\eta(x, y)=[I-\mathcal{K}]^{-1} u(x, y)=\sum_{\ell=0}^{\infty} \mathcal{K}^{\ell} u(x, y) .
$$

Clearly, $\eta$ is determined in terms of the arbitrary functions $u_{ \pm}$.

For $v=0$, i.e., a free particle, $\eta(x, y)=u(x, y)$. As shown in Ref. 9, this is equivalent to

$$
\eta=L(p)+K(p) \mathcal{P},
$$

where $L(p)^{\dagger}=L(p)$ and $K(p)^{\dagger}=\mathcal{P} K(p) \mathcal{P}$, equivalently $L$ and $K$ are respectively real-valued and $\mathcal{P} \mathcal{T}$-invariant [this means $K(r)^{*}=K(-r)$ for all $r \in \mathbb{R}$ ] functions. (They may be further restricted to constants if one postulates the nonexistence of a hidden length scale for the problem. See Ref. 9 for details.) They are related to the Fourier transform [in our convention, the Fourier transform of a function $\varphi$ is given by $\left.\tilde{\varphi}(k):=(2 \pi)^{-1 / 2} \int_{-\infty}^{\infty} \mathrm{d} x e^{-i k x} \varphi(x)\right] \tilde{u}_{ \pm}$of $u_{ \pm}$according to 


$$
L(p)=\sqrt{2 \pi} \tilde{u}_{+}\left(\frac{p}{\hbar}\right), \quad K(p)=\sqrt{2 \pi} \tilde{u}_{-}\left(-\frac{p}{\hbar}\right) .
$$

For a real-valued potential the ordinary choice for the metric operator that yields the $L^{2}$-inner product, i.e., $\eta=I$, corresponds to setting

$$
u(x, y)=\delta(x-y)-\frac{m}{\hbar^{2}} \int^{x+y / 2} \mathrm{~d} r v(r) .
$$

To see this, we first calculate $K \delta(x-y)$ for an arbitrary (possibly complex-valued) potential $v$. Using the well-known properties of the step function

$$
\theta(x):=\left\{\begin{array}{cc}
0 & \text { for } x<0 \\
\frac{1}{2} & \text { for } x=0 \\
1 & \text { for } x>0
\end{array}\right.
$$

we then find

$$
\mathcal{K} \delta(x-y)=\frac{m}{\hbar^{2}}\left(\int^{x+y / 2} \mathrm{~d} r \Re[v(r)]+i \operatorname{sign}(y-x) \int^{x+y / 2} \mathrm{~d} r \Im[v(r)]\right),
$$

where $\mathfrak{R}[v]$ and $\Im[v]$ respectively stand for the real and imaginary parts of $v$, and

$$
\operatorname{sign}(x):=\theta(x)-\theta(-x)=\left\{\begin{array}{cc}
-1 & \text { for } x<0 \\
0 & \text { for } x=0 \\
1 & \text { for } x>0
\end{array}\right.
$$

If $v$ is a real potential, $\mathfrak{R}[v]=v$ and $\Im[v]=0$. In this case (26) together with (19) and $\eta(x-y)$ $=\delta(x-y)$ yield $(24)$.

For a purely imaginary potential, $\mathfrak{R}[v]=0, v=i \Im[v]$, and (26) takes the following form:

$$
\mathcal{K} \delta(x-y)=\frac{m}{\hbar^{2}} \operatorname{sign}(y-x) \int^{x+y / 2} \mathrm{~d} r v(r) .
$$

\section{APPLICATIONS}

\section{A. $\mathcal{P} \mathcal{T}$-symmetric square well}

The $\mathcal{P} \mathcal{T}$-symmetric square well potential,

$$
v(x):= \begin{cases}-i \zeta \operatorname{sign}(x) & \text { for }|x|<\frac{L}{2} \\ \infty & \text { for }|x|>\frac{L}{2}\end{cases}
$$

with $\zeta \in \mathbb{R}$ and $L \in \mathbb{R}^{+}$, defines one of the best-known exactly solvable toy models that captures the generic properties of pseudo-Hermitian quantum systems. ${ }^{24,25}$ A thorough investigation of the physical content of this model is conducted in Ref. 4, where a particular perturbative calculation of a metric operator and the corresponding physical observables, localized states, probability density, and the underlying classical Hamiltonian is performed. This calculation makes use of the fact that the non-Hermiticity effects in this model diminish for energy states with larger spectral label $N$. More specifically, it is $\zeta / N^{2}$ that plays the role of the perturbation parameter.

More recently, Bender and $\operatorname{Tan}^{12}$ performed a more conventional perturbative calculation of a metric operator taking $\zeta$ as the perturbation parameter. This is the metric operator $\eta_{+}$that is 
associated with the $\mathcal{C P} \mathcal{T}$-inner product $(\cdot, \cdot)_{\mathcal{C P T}},{ }^{26}$ that is $(\cdot, \cdot)_{\mathcal{C P T}}=\left\langle\cdot \mid \eta_{+} \cdot\right\rangle \cdot{ }^{21}$ Expressing $\eta_{+}$in its exponential form, $\eta_{+}=e^{-Q}$, and noting that Bender and Tan set $\hbar=2 m=L / \pi=1$, take $\epsilon=-\zeta$ for the coupling constant, and use " $\varepsilon(x)$ " for "sign $(x)$," we can summarize their principal result [Eq. (11) of Ref. 12] as

$$
\langle x|Q| y\rangle=: Q(x, y)=-\frac{i \zeta}{4}[x-y+\operatorname{sign}(x-y)(|x+y|-\pi)]+\mathcal{O}\left(\zeta^{3}\right),
$$

where $\mathcal{O}\left(\zeta^{n}\right)$ stands for terms of order $n$ and higher in powers of $\zeta$. In particular, in view of the identity $x-y=|x-y| \operatorname{sign}(x-y)$, we have the following expression for the $\mathcal{C P} \mathcal{T}$-metric operator $\eta_{+}$:

$$
\eta_{+}(x, y)=\delta(x-y)+\frac{i \zeta}{4}(|x-y|+|x+y|-\pi) \operatorname{sign}(x-y)+\mathcal{O}\left(\zeta^{2}\right) .
$$

The perturbative calculation of the metric operator using the method developed in the preceding section is quite straightforward. Inserting (28) in (27) and performing the trivial integral in the resulting equation, we find

$$
\mathcal{K} \delta(x-y)=\frac{i m \zeta}{2 \hbar^{2}}|x+y| \operatorname{sign}(x-y) .
$$

The most general metric operator $\eta$ that reduces to the identity operator in the Hermitian limit $\zeta \rightarrow 0$ is obtained by setting

$$
u(x, y)=\delta(x-y)+\zeta\left[w_{+}(x-y)+w_{-}(x+y)\right]+\mathcal{O}\left(\zeta^{2}\right)
$$

in (21), where $w_{ \pm}:[-L / 2, L / 2] \rightarrow \mathrm{C}$ are arbitrary functions satisfying $w_{ \pm}(x)^{*}=w_{ \pm}(\mp x)$ and $w_{ \pm}( \pm L)=0$. [These conditions arise from the Hermiticity requirement on the metric operator and its spectral resolution (8).] This together with (31) yields

$$
\eta(x, y)=\delta(x-y)+\zeta\left[w_{+}(x-y)+w_{-}(x+y)+\frac{i m}{2 \hbar^{2}}|x+y| \operatorname{sign}(x-y)\right]+\mathcal{O}\left(\zeta^{2}\right) .
$$

Setting $\hbar=2 m=L / \pi=1$ in this equation, we find that the $\mathcal{C P} \mathcal{T}$-metric operator (30) obtained by Bender and $\operatorname{Tan}^{12}$ is a particular example of the metric operators (33). It corresponds to the choice $w_{+}(x)=(i / 4)(|x|-\pi) \operatorname{sign}(x)$ and $w_{-}(x)=0$.

We can calculate higher-order terms in the expression for the metric operator using our iterative method. Each additional order will involve an arbitrary pair of functions that enter the expression for $u$ in (21). This calculation is not only completely general (as it yields the most general metric operator), but it is also much simpler to perform. This is mainly because unlike its alternatives $^{4,12}$ it avoids approximating or summing complicated series.

\section{B. An imaginary scattering potential}

Consider the following variant of the $\mathcal{P} \mathcal{T}$-symmetric square well potential: ${ }^{27}$

$$
v(x):=\frac{i \zeta}{2}\left[\operatorname{sign}\left(x+\frac{L}{2}\right)+\operatorname{sign}\left(x-\frac{L}{2}\right)-2 \operatorname{sign}(x)\right]= \begin{cases}-i \zeta \operatorname{sign}(x) & \text { for }|x|<\frac{L}{2} \\ 0 & \text { for }|x|>\frac{L}{2},\end{cases}
$$

where $\zeta \in \mathbb{R}$ is a coupling constant and $L \in \mathbb{R}^{+}$is a length scale.

In Ref. 7 we established the reality of the spectrum of this potential and used the spectral method of Refs. 14, 17, and 18 to obtain a perturbative expression for an associated metric operator $\eta_{+}$. This involved constructing an appropriate biorthonormal system for the model and performing a highly tedious calculation of the integrals appearing in the spectral resolution of $\eta_{+}$. 
Indeed, this calculation could only be done after expanding all the relevant quantities in powers of $\zeta$ and restricting to the first-order terms. Although the results reported in Ref. 7 required performing extremely lengthy calculations partly done using Mathematica, the expression obtained for $\eta_{+}(x, y):=\left\langle x\left|\eta_{+}\right| y\right\rangle$ took a surprising simple form, namely

$$
\eta_{+}(x, y)=\delta(x-y)+\frac{i m \zeta}{4 \hbar^{2}}(2 L+2|x+y|-|x+y+L|-|x+y-L|) \operatorname{sign}(x-y)+\mathcal{O}\left(\zeta^{2}\right) .
$$

Here we wish to use the scheme developed in the preceding section to construct the most general metric operator $\eta$ that reduces to the identity operator in the Hermitian limit $\zeta \rightarrow 0$. In order to do this first we insert (34) in (27) and perform the trivial integral in the resulting equation to obtain

$$
\mathcal{K} \delta(x-y)=\frac{i m \zeta}{4 \hbar^{2}}(|x+y+L|+|x+y-L|-2|x+y|) \operatorname{sign}(y-x) .
$$

Substituting (32) in (21) and using (36), we then find

$$
\begin{aligned}
\eta(x, y)= & \delta(x-y)+\zeta\left[w_{+}(x-y)+w_{-}(x+y)+\frac{i m}{4 \hbar^{2}}(2|x+y|-|x+y+L|-|x+y-L|) \operatorname{sign}(x-y)\right] \\
& +\mathcal{O}\left(\zeta^{2}\right),
\end{aligned}
$$

where $w_{ \pm}: \mathbb{R} \rightarrow \mathrm{C}$ are arbitrary functions satisfying $w_{ \pm}^{*}(x)=w_{ \pm}( \pm x)$. Clearly, the positive-definite inner product (35) constructed in Ref. 7 corresponds to setting $w_{+}(x)=i m L / 2 \hbar^{2} \operatorname{sign}(x)$ and $w_{-}(x)=0$.

\section{Imaginary $\delta$-function potentials}

Consider the potential

$$
v(x)=i \zeta \delta(x-a),
$$

where $\zeta, a \in \mathrm{R}$. (Clearly, we can choose the origin of the $x$-axis so that $a=0$. We retain $a$ for future use where we consider the multi-delta-function potentials.) It is not difficult to solve the timeindependent Schrödinger equation for this potential and show that $H=\left(p^{2} / 2 m\right)+v(x)$ has a real continuous spectrum. $[H$ is not $\mathcal{P} \mathcal{T}$-symmetric. But one may attempt to use the results of Ref. 21 to construct a generalized $\mathcal{P} \mathcal{T}$-operator (an antilinear involution) that commutes with $H$.] This in turn suggests that one can construct an associated pseudo-metric operator using the spectral method of Refs. 17 and 18. This construction is similar to the one offered in Ref. 7 for the potential (34). An explicit calculation of $\eta$ using this method is however quite involved. A much simpler construction that we will describe in the following is based on the method of Sec. III.

First, we substitute (38) in (20) to establish

$$
\mathcal{K} F(x, y)=\mathcal{F}(x, y)+\mathcal{F}(y, x)^{*}, \quad \mathcal{F}(x, y):=\frac{i z}{2} \theta(y-a) \int_{x-y+a}^{x+y-a} \mathrm{~d} s F(s, a),
$$

where $z:=2 m \zeta / \hbar^{2}$ and $F(x, y)$ is a test function. If we choose a $z$-independent $u$, the series expansion (21) becomes a power series in the coupling constant $z$. For definiteness we shall first choose $u(x, y)=\delta(x-y)$. Setting $F(x, y)=\delta(x-y)$ in (39) and using the properties of the step function (25), we then find

$$
\mathcal{K} u(x, y)=\frac{i z}{2} \theta(x+y-2 a) \operatorname{sign}(y-x)=: u_{1}(x, y) .
$$

Alternatively, we could directly use (27) to obtain (40).

Next, we compute $\mathcal{K}^{2} u(x, y)$ by substituting $u_{1}$ for $F$ in (39). This yields 


$$
\mathcal{K}^{2} u(x, y)=\frac{z^{2}}{4}[\theta(x-a)+\theta(y-a)][(x+y-2 a) \theta(x+y-2 a)-|x-y|] .
$$

The higher-order terms in (21) can be similarly calculated. Moreover, because of the simple form of (39) and (40), we can actually obtain an upper bound on $\left|\mathcal{K}^{\ell} u(x, y)\right|$ and use it to find a lower bound on the radius of the convergence of the series (21).

First, we recall ${ }^{28}$ that if a function $g: \mathrm{R} \rightarrow \mathrm{C}$ is bounded on an interval $[\alpha, \beta]$ by some $M$ $\in \mathbb{R}^{+}$, i.e., $|g(r)|<M$ for all $r \in[\alpha, \beta]$, then $\left|\int_{\alpha}^{\beta} \mathrm{d} r g(r)\right| \leqslant M(\beta-\alpha)$. Now, let $F(x, y)$ be a function such that $|F(s, a)|$ has an upper bound $M_{F}$ as $s$ takes values between $|x-y|+a$ and $x+y-a$. Then, according to (39),

$$
|\mathcal{K} F(x, y)| \leqslant|z|(|x-a|+|y-a|) M_{F} .
$$

In view of (40), for all $x, y \in \mathbb{R},|\mathcal{K} u(x, y)| \leqslant|z| / 2$. To obtain an upper bound on $\left|\mathcal{K}^{2} u(x, y)\right|$ we set $F(x, y)=\mathcal{K} u(x, y)$ in (42), which allows us to identify $M_{F}$ with $|z| / 2$ and yields for all $x, y \in \mathbb{R}$ :

$$
\left|\mathcal{K}^{2} u(x, y)\right| \leqslant \frac{z^{2}}{2}(|x-a|+|y-a|)
$$

We can directly verify this relation using (41). Repeating the procedure that leads to (43), we find for all $\ell \geqslant 1$ and all $x, y \in \mathbb{R}:\left|\mathcal{K}^{\ell} u(x, y)\right| \leqslant|z|^{\ell}(|x-a|+|y-a|)^{\ell-1} / 2$. This in turn implies, in view of the elementary comparison tests, that the series (21) converges (absolutely) for $|z|(|x-a|+|y-a|)$ $<1$. Hence, it converges in an open disc in the $x-y$ plane that is centered at $(x=a, y=a)$ and has a radius $\varrho>(\sqrt{2}|z|)^{-1}$.

In summary, for every given value of $z$, truncation of the series (21) yields a reliable approximation for $\eta$ provided that we keep a sufficiently large number of terms in the series and deal with wave functions $\psi(x)$ that decay sufficiently rapidly as $|x| \rightarrow \infty$. (Our analysis only yields a lower bound on $\varrho$. It does not imply that $\varrho$ is finite.)

We can extend our treatment to a potential consisting of more than one delta function:

$$
v(x)=i \sum_{n=1}^{N} \zeta_{n} \delta\left(x-a_{n}\right)
$$

where $N \in \mathbb{Z}^{+}$and $\zeta_{n}, a_{n} \in \mathbb{R}$. An example is the $\mathcal{P} \mathcal{T}$-symmetric potentials ${ }^{29-31}$ corresponding to the cases that $N$ is even and $\zeta_{N / 2+k}=-\zeta_{k}, a_{(N / 2)+k}=-a_{k}$ for all $k=1,2, \cdots N / 2$. [Here we assume that $\left(\zeta_{n}, a_{n}\right)$ are such that the Hamiltonian is pseudo-Hermitian. This is the generic case, for the values of $\left(\zeta_{n}, a_{n}\right)$ that render the spectrum of the Hamiltonian nonreal form a measure-zero subset of the set $\mathbb{R}^{2 N}$ of all possible values of $\left(\zeta_{n}, a_{n}\right)$.]

For these multi-delta-function potentials the calculation of the first-order term in $z_{n}$ $:=2 m \zeta_{n} / \hbar^{2}$ in the series expansion (21) reduces to the case $N=1$ that we considered above. In view of (40),

$$
\mathcal{K} \delta(x, y)=\frac{i}{2} \sum_{n=1}^{N} z_{n} \theta\left(x+y-2 a_{n}\right) \operatorname{sign}(y-x) .
$$

The most general $\eta$ that reduces to $\eta=I$ in the Hermitian limit $z_{n} \rightarrow 0$ is obtained up to secondorder terms in $z_{n}$ by setting $u(x, y)=\delta(x-y)+\sum_{n=1}^{N} z_{n}\left[w_{n+}(x-y)+w_{n-}(x+y)\right]+\mathcal{O}\left(z_{n}^{2}\right)$, where $w_{n \pm}: \mathrm{R} \rightarrow \mathrm{C}$ are arbitrary functions satisfying $w_{n \pm}(x)^{*}=w_{n \pm}(\mp x)$. This together with (45) and (21) yields 


$$
\eta(x, y)=\delta(x-y)+\sum_{n=1}^{N} z_{n}\left[w_{n+}(x-y)+w_{n-}(x+y)+\frac{i}{2} \theta\left(x+y-2 a_{n}\right) \operatorname{sign}(y-x)\right]+\mathcal{O}\left(z_{n}^{2}\right) .
$$

We close this section by the following general remarks. As we observe in the study of the above toy models, the series solution (21) may be used to obtain a perturbative expansion for the pseudo-metric operator $\eta$. In general, depending on the details of the model under study, one may or may not have access to a dimensionless perturbation parameter. Typical examples for which this occurs are the imaginary cubic potential and the single imaginary delta-function potential (38). In this case, as explained in Ref. 9, the truncation of the perturbative expansion of $\eta(x, y)$ generally yields a reliable result only within a sufficiently small region in the $x-y$ plane. Furthermore, one expects that for sufficiently small values of the perturbation parameter (the coupling constant $\zeta$ or $\zeta_{n}$ in the above examples) the perturbative corrections to a positive-definite metric operator such as $\eta=I$ leave this property intact.

\section{CONCLUDING REMARKS}

In this article, we have outlined a differential realization of the pseudo-Hermiticity condition that plays a central role in devising a unitary quantum theory based on quasi-Hermitian Hamiltonians of the standard form. The integral kernel $\eta(x, y)$ for the corresponding pseudo-metric operators $\eta$ satisfies a linear partial differential equation. For systems having $\mathrm{R}$ as their configuration space this is nothing but a particular variable-mass Klein-Gordon equation. We have obtained a general series solution for this equation and demonstrated its application in treating the $\mathcal{P} \mathcal{T}$-symmetric square well potential, an imaginary $\mathcal{P} \mathcal{T}$-symmetric scattering potential, and a class of imaginary delta-function potentials. In particular, for the former two potentials, the approach presented here is by far more practical than the alternative approaches that use the spectral resolution of the metric operator. Another advantage of the former approach is that it is capable of producing the most general pseudo-metric operator. In particular, imposing the positivedefiniteness condition (7), it yields the general form of the metric operators.

Our method is not only practically advantageous but also conceptually appealing. It furthers the analogy between QM and GR, for the differential pseudo-Hermiticity relation plays a similar role in QM as the Einstein's field equation does in GR [not to mention the curious fact that the field theoretic extension of the pseudo-Hermiticity relation (3) is a functional differential equation that has the same structure as the Wheeler-DeWitt equation of the conventional canonical quantum gravity]. Another valuable outcome of our method is a concrete characterization of the arbitrariness of the metric operator. Each choice of a metric operator defines a separate quantum system. One can pursue the prescription used in the so-called quasi-Hermitian quantum mechanics ${ }^{1}$ to select an irreducible set of compatible quasi-Hermitian operators $O_{\alpha}$ and fix the metric operator $\eta_{+}$ (up to scale) through the requirement that $O_{\alpha}$ be $\eta_{+}$-pseudo-Hermitian. Alternatively, one can follow the approach of the so-called pseudo-Hermitian quantum mechanics, ${ }^{3}$ choose $\eta_{+}$directly, and construct the Hilbert space and observables of the theory accordingly., ${ }^{4,7,9}$

${ }^{1}$ F. G. Scholtz, H. B. Geyer, and F. J. W. Hahne, Ann. Phys. (N.Y.) 213, 74 (1992).

${ }^{2}$ A. Mostafazadeh, Phys. Lett. A 320, 375 (2004).

${ }^{3}$ A. Mostafazadeh, Czech. J. Phys. 53, 1079 (2003).

${ }^{4}$ A. Mostafazadeh and A. Batal, J. Phys. A 37, 11645 (2004).

${ }^{5}$ A. Mostafazadeh, J. Phys. A 38, 6557 and 8185 (2005).

${ }^{6}$ H. F. Jones, J. Phys. A 38, 1741 (2005).

${ }^{7}$ A. Mostafazadeh, J. Math. Phys. 46, 102108 (2005).

${ }^{8}$ B. Bagchi, C. Quesne, and R. Roychoudhury, J. Phys. A 39, L127 (2006).

${ }^{9}$ A. Mostafazadeh, eprint: quant-ph/0508195, J. Phys. A (to be published).

${ }^{10}$ F. G. Scholtz and H. B. Geyer, Phys. Lett. B 634, 84 (2006).

${ }^{11}$ C. M. Bender and G. V. Dunne, Phys. Rev. D 40, 2739 (1989); 40, 3504 (1989).

${ }^{12}$ C. M. Bender and B. Tan, J. Phys. A 39, 1945 (2006).

${ }^{13}$ H. F. Jones and J. Mateo, Phys. Rev. D 73, 085002 (2006).

${ }^{14}$ A. Mostafazadeh, J. Math. Phys. 43, 205 (2002). 
${ }^{15}$ T. Kato, Perturbation Theory for Linear Operators (Springer, Berlin, 1995).

${ }^{16}$ W. Pauli, Rev. Mod. Phys. 15, 175 (1943).

${ }^{17}$ A. Mostafazadeh, J. Math. Phys. 43, 2814 (2002).

${ }^{18}$ A. Mostafazadeh, J. Math. Phys. 43, 3944 (2002).

${ }^{19}$ A. Mostafazadeh, eprint quant-ph/0310164.

${ }^{20}$ A. Mostafazadeh, Nucl. Phys. B 640, 419 (2002).

${ }^{21}$ A. Mostafazadeh, J. Math. Phys. 44, 974 (2003).

${ }^{22}$ M. Tegmark, Class. Quantum Grav. 14, L69 (1997).

${ }^{23}$ A. Bohm, Quantum Mechanics: Foundations and Applications, Vol. 3 (Springer, Berlin, 1993).

${ }^{24}$ M. Znojil, Phys. Lett. A 285, 7 (2001).

${ }^{25}$ B. Bagchi, S. Mallik, and C. Quesne, Mod. Phys. Lett. A 17, 1651 (2002).

${ }^{26}$ C. M. Bender, D. C. Brody, and H. F. Jones, Phys. Rev. Lett. 89, 270401 (2002).

${ }^{27}$ A. Ruschhaupt, F. Delgado, and J. G. Muga, J. Phys. A 38, L171 (2005).

${ }^{28}$ J. M. Howie, Complex Analysis (Springer, London, 2003).

${ }^{29}$ S. Albeverio, A.-M. Fei, and P. Kurasov, Lett. Math. Phys. 59, 227 (2002).

${ }^{30}$ M. Znojil and V. Jakubski, J. Phys. A 38, 5041 (2005); Czech. J. Phys. 55, 1113 (2005).

${ }^{31}$ E. Demiralp, Czech. J. Phys. 55, 1081 (2005). 